\title{
El circulo de la palabra, tecnología ancestral e intercultural en la comunidad Yanakuna -Popayán Cauca
}

\author{
The circle of word, ancestral and intercultural technology in the \\ Yanakuna community - Popayán Cauca
}

Over Hernán Majín Melenje ${ }^{1}$

\section{Resumen}

El círculo de la palabra es un espacio de vida ancestral que ha hecho parte del proceso colectivo Yanakuna. La tullpa (fogón) ha sido, es y será un camino en donde día a día se aprenden y se comparten sabidurías y conocimientos. Al llegar la modernidad, la vida "civilizada" fragmentó estas formas y modos de establecer el orden de la vida en familia, y se definieron otras reglas que poco a poco destruyeron los caminos del pensamiento propio. El cultivo y crianza de sabidurías desde el círculo de la palabra Yanakuna es un camino de esperanza, en donde las nuevas semillas germinarán y con sus frutos llegarán a otros espacios aportando saberes propios sin importar la época ni el contexto. En este caminar hacia el sumak kawsay o buen vivir se puso en práctica la realización de círculos de palabra Yanakuna como tecnología ancestral para revitalizar los saberes ancestrales que se han debilitado en un contexto de ciudad como Popayán-Cauca. Es importante afirmar que uno de los principales resultados en este CCRISAC ha sido la voluntad de la comunidad por caminar hacia el retorno de lo ancestral y, evidenciar, logros significativos en el compartir de la palabra y el amor a la vida yanakuna. Se puede concluir, que hoy, pese a los espacios de modernidad en los que vivimos, es posible poner en prácticas los saberes ancestrales que revitalizan la historia y el legado de los mayores y mayoras de nuestro pueblo Yanakuna.

Palabras clave: Círculo de la palabra; yanakuna; ancestralidad; interculturalidad.

\section{Abstract}

The circle of word is an ancestral space of life that has been part of the Yanakuna collective process. The tullpa (stove) has been, is and will be a path where day by day it is learnt and shared the wisdom and knowledge. When modernity arrived, the "civilized" life fragmented these forms and ways of establishing the order of family life, and other rules were defined that little by little destroyed the paths of our own thinking. The cultivation and wisdom upbringing from the circle of the Yanakuna word is a path of hope, where the new seeds will germinate and with their fruits

1 Máster en Comunicación Intercultural con Enfoque en Género. Email:chasktnoticias@gmail.com

Recibido: 26/02/2018 Aprobado: 28/08/2018 
they will reach other spaces contributing their own knowledge regardless of time or context. In this journey towards sumak kawsay or good living, the implementation of Yanakuna word circles was put into practice as an ancestral technology to revitalize the ancestral knowledge that has weakened in a city context such as Popayán-Cauca. It is important to affirm that one of the main results in this CCRISAC has been the community's willingness to walk towards the return of the ancestral and, evidencing, significant achievements in sharing the word and the love of Yanakuna life. It can be concluded that today, despite the spaces of modernity in which we live, it is possible to put into practice the ancestral knowledge that revitalizes the history and the legacy of the elders of our Yanakuna people.

Keywords: Circle of word; Yanakuna; ancestry; interculturality.

\section{Introducción}

El círculo de la palabra, la tecnología ancestral e intercultural en la comunidad Yanakuna -Popayán Cauca es un proceso descolonizante para abordar el cultivo y crianza de sabidurías y conocimientos en un contexto de ciudad, espacio donde se desarrolló con comuneros yanakuna que se interesan por una vida distinta, $y$, un espacio para aprender de los mayores y mayoras.

Se eligió un cultivo y crianza de sabidurías y conocimientos "CCRISAC" tomando en cuenta que en la ciudad de Popayán se tiene un proceso de más de veinte años de vida urbana Yanakuna, hoy uno de los temores es que el saber que se ha mantenido pueda debilitarse, incluso perderse y quedar una generación de indígenas sin un soñar, un sentir y un hacer como Yanakuna. La línea escogida dentro del CCRISAC fue Sistemas y procesos de comunicación propios en nacionalidades, pueblos y comunidades de Abyayala.

Este CCRISAC se abordó teniendo en cuenta las normas que se aportan desde la sabiduría ancestral, acuñados en la actualidad como normas de cultivo y crianza de sabidurías y conocimientos. La Chakana es una de las formas que nos dieron las guías para podernos orientar, así mismo en ese ejercicio poder descubrir que la palabra CCRISAC tiene siete letras, las he relacionado con los siete colores del arco iris o kuichi, y no es casualidad, ni coincidencia, es el orden que la misma palabra mayor hizo que se diera vida a esta palabra. En este sentido, la Chakana es el orden, la guía y espacio que dio la ruta para abordar la investigación de este CCRISAC. (Cabildo Mayor del Pueblo Yanacona 2012).

Los productos obtenidos de este bonito cultivo y crianza de sabidurías y conocimientos fue encontrar unos llamativos espacios de vida que día a día dan vitalidad al Yanakuna como: la tullpa, memoria oral, simbología ancestral, lenguajes de la madre tierra, espiritualidad propia, el caminar de la Palabra y Kapak ñan (gran camino Inka). 
Asimismo, en este ejercicio conocer a través de los relatos y entrevistas un sinnúmero de aportes valiosos que no se han escrito, pues están en la memoria oral de cada mayor y mayora de nuestro pueblo Yanakuna.

El plan fue abordar siete círculos de la palabra con los siete temas y cada tema con un color del arco iris, una bonita experiencia que hoy dejamos a nuestros compañeros para que se siga investigando y revitalizando, el saber, nunca termina, es inacabado, pues siempre se aprenderá donde quiera que vayamos.

Las distintas temáticas se abordaron principalmente en la ciudad y en otros espacios externos para vivenciar la vida Yanakuna y unos en especial en territorios ancestrales para darle más vida al proceso del caminar de la palabra a través de la unidad y el círculo de unidad.

Respecto a delimitar los antecedentes bibliográficos es importante expresar que este CCRISAC en su esencia es el resultado de la reflexión de nuestro caminar, pues son casi invisibles los estudios que hayan buscado preguntar por la vida yanakuna desde un estudio científico, es decir es la palabra mayor la que prevalece, la medicina ancestral y mensaje del abuelo Yagé, la coca y el mismo caminar a los sitios sagrados. El círculo de la palabra por lo tanto no es estático, es itinerante, siempre se estará atizonando el fogón para que la llama de espiritualidad Yanakuna no se apague.

\section{Revisión de literatura}

Este cultivo y crianza de sabidurías y conocimientos se desarrolló en la comunidad Yanakuna, un término de la lengua Runa Shimi (Kichwa) de origen Inca. Se dice que el Yanakuna es de origen del Perú y su nombre proviene de una región llamada Yanayaku (Agua Negra: Yana: negro y Yaku: agua). También significa "gente de la noche, de la oscuridad, del cosmos, del más allá". Al Yanakuna se le conoce como una persona pensante, y su relación con la noche es debido a que es reflexivo y pacífico. (Pensamiento Colectivo Yanakuna, 2014)

Es importante resaltar que desde la cosmovisión andina existen muchas formas propias de investigación y aprendizaje; estas son las vivencias, las prácticas, la observación, el silencio, la medicina, la decodificación de los lenguajes de los seres de la madre tierra y el cosmos. En el caso de los pueblos andinos esta la Chakana, una estrategia que complementa el proceso de cultivar y criar, sabidurías y conocimientos.

Actualmente, desde la Red de Universidades Indígenas, Interculturales y Comunitarias del Abya Yala (RUIICAY) se establecen caminos con ideas descolonizantes y mucha sabiduría desde países inmersos en continuas reflexiones sobre los procesos de investigación propios como una alternativa para trascender como pueblos indígenas en tiempos de modernidad. 
En el proceso Yanakuna a nivel de Colombia se establece desde hace más de 20 años un direccionamiento de adelantar un trabajo en equipo con las comunidades a nivel nacional, se han ubicado en seis departamentos del país, entre ellos: Cauca, Valle del Cauca, Quindío, Huila, Putumayo y Cundinamarca (Bogotá D.C)

La dinámica de establecer una propuesta de un Sistema Educativo Indígena Propio Yanacona - SEPIY ha dado frutos interesantes, uno de ellos el florecimiento de la identidad cultural en donde el idioma Runa Shimi vuelve a surgir y así mismo otros procesos como la minga, el caminar juntos, la simbología propia y espiritualidad.

Frente al sueño de este camino de avanzar en la comunidad Yanakuna de contexto en la cuidad en Popayán, implica que hay que volver a sembrar el conocimiento con el acompañamiento de los mayores y mayaras Yanaconas del cabildo, cultivarlo así mismo en los niños y jóvenes, es una de las formas para evitar que los procesos comunicacionales se debiliten. Somos conscientes que en las ciudades cada día existe influencia de los medios externos, que hacen perder la identidad y por ello es una tarea como investigadores interculturales es avanzar en la búsqueda de espacios que motiven a la comunidad Yanacona a volver a caminar el proceso de lucha y resistencia.

Frente a los saberes de los pueblos originarios de América Latina es muy poco lo que se ha escrito frente a los conceptos propios sobre determinados procesos colectivos de los pueblos, es así que a continuación primeramente se busca conceptualizar algunos pensamientos que se han recopilado del pensar integral, en este caso para el pueblo Yanacona en particular, sin embargo existen algunos trabajos que se han realizados en universidades convencionales como la Nacional de Colombia, Universidad de Antioquia y en este espacio resaltaremos algunos conceptos sin antes conocer los fundamentos propios desde la visión Yanacona.

Los círculos de palabra en el contexto Yanacona han sido espacios de interacción a través de los tiempos, las formas circulares de dialogo han permitido a través de la historia demostrar una forma armónica del compartir sabidurías y conocimientos con humildad, es así que se demuestra que ha existido la complementariedad, reciprocidad, armonía y respeto único de unos a otros, esto demuestra un dialogo a niveles de igualdad, pues el conversar en forma circular le permite a los yanaconas poder observar y vivenciar la historia de vida. (Pensamiento colectivo Yanacona).

Se encuentran algunas apreciaciones aisladas en donde se afirma que:

"El circulo de palabra es una tradición milenaria utilizada por los Muiscas (Pueblo Indígena del altiplano Cundiboyacence de Colombia). Este ritual se destaca por el respeto a la Madre Tierra y la relación con los astros, la unión de la creación donde la espiritualidad y el concepto de humanidad prevalecen sobre los conceptos individuales. Esta sabiduría ancestral concibe el territorio como un lugar sagrado donde interactúa 
con la naturaleza y los animales en un sentido profundamente integrador. Todo está unido en el universo conjuntamente con los seres de la Tierra y donde tienen gran importancia las plantas sagradas, la relación directa de la Pacha Mama”. (Achury 2015).

A través del juego "Círculos de la palabra" se logra trasportar un conocimiento local a otro espacio, a otra realidad, materializando una cultura con la vivencia de que sus tejidos, objetos, trajes, técnicas, materiales sobrevivan con toda su carga simbólica (Agencia de Noticias, UNC, 12 abril del 2016). Los círculos sagrado son en silencio, devoción, oración o cánticos.

Latinoamérica es un continente de tradición oral. La cultura de los distintos pueblos que crearon y conformaron este rico continente, ha sido transmitida mediante la palabra y de generación en generación. La oralidad se ha ido enriqueciendo y cambiando de forma permanente, ya sea por medio de la escritura o, a través de los medios de comunicación masiva.

Durante siglos, las antiguas culturas se reunían en torno a un relator que, haciendo uso de la palabra hablada, transmitía las costumbres, creencias ancestrales y tradiciones de todo un pueblo. La vida cotidiana, social, religiosa y económica era transmitido de manera oral, mediante relatos históricos que se iban inculcando de generación en generación. Estos conformaban la identidad cultural y la cohesión del grupo social.

Los indígenas no sabían leer ni escribir, y la palabra era el instrumento más importante y clave en la formación de los pueblos y Latinoamérica. Cada pueblo tenía una tradición acorde a sus necesidades históricas y sociales particulares. (Oralidad en Latinoamérica, 2010). Esta oralidad, surgió de la necesidad del ser humano para explicar los fenómenos naturales y sociales. Es la doctrina más antigua de la que se sirvieron los originarios, para entenderse unos con otros y lograr la unión e identidad propia.

Respecto a los indígenas yanaconas es importante expresar que la memoria ancestral se ha venido pasando de generación en generación, utilizando espacios de relacionamiento en actividades cotidianas del diario vivir como el trabajo de la chakra, en la elaboración de los tejidos, participación en ritualidades andinas como los inti Raymi , Killa Raymi entre otros espacios de vida armónica.

\section{Cosmovisión, usos y costumbres del Pueblo Yanakuna}

El pueblo Yanacona es muy rico en el campo de la espiritualidad, a continuación retomando elementos de la página de la nación Yanakuna, se busca dar un sentido del ideal que se busca apoyar y fortalecer desde los círculos de la palabra.

Todos estos fundamentos de la cosmovisión Yanacona, hoy se pueden conocer gracias al proceso del trabajo de las autoridades Yanaconas, hoy agrupadas en el 
Cabildo Mayor del pueblo Yanacona, dentro de este ejercicio es importante retomar estos fundamentos para poder dar a entender el trabajo de investigación que busca dinamizar campos de trabajo comunitario para que en el contexto urbano de Popayán se pueda caminar en el tan esperado buen vivir.

Para poder acercarnos al proceso de esta investigación considero de suma importancia el poder documentarse de los avances que se han realizado en el campo de la sistematización de nuestro pueblo Yanakuna respecto al campo de los conocimientos propios, aquí se puede evidenciar parte de ello, resaltando que ya se han publicado varias memorias que hoy contribuyen a este camino del camino hacia el Sumak Hatun ñan: Bonito camino de sabiduría.

El Sumak Kaway Hatun Ñan (El camino hacia el buen vivir) es el resultado de retomar los procesos del idioma Runa Shimi (Kichwa) en el pueblo Yanakuna desde hace ya más de 10 años. En el 2002 llegan caminantes a la región provenientes de Ecuador, Bolivia, Perú, norte de Argentina y siembran en nuestro proceso esa chispa de encaminar el proceso hacia de idioma. De allí surge dentro del proceso de espiritualidad propia recorrer los caminos ancestrales, caminos como el paso al Huila por el páramo del Letrero, ese ejercicio permitió revitalizar la memoria y denominarle a esos y muchos otros espacios como son los caminos riales (caminos que construyeron los indígenas y luego los españoles les dieron el nombre de camino real) como se les llamaba por los mayores. Se les da otra connotación en idioma Kichwa como Sumak Kaway Hatun Ñan.

Hoy este concepto ha ido tomando fuerza y se establece dentro de la filosofía propia como un espacio para volver al camino que caminaron nuestros mayores y mayoras, es también un camino para volver a la medicina, al tejido propio, a los conversatorios antiguos, como por ejemplo enterrar de nuevo tres Tullpas (fogón) para conversar alrededor del Inti Taita (padre fuego)

A continuación se busca abordar las distintas formas y modos de espacios en donde los mayores y mayoras han establecido en sus vivencias propias de unidad compartiendo saberes, así mismo donde se han cultivado y sembrado sabidurías y conocimientos. Dentro de este espacio de los procesos de construcción permanente de sabidurías y conocimientos desde los círculos de la palabra que se vivencian es muy importante plasmar a continuación las formas de CCRISAC que siguen dando vida al territorio Yanakuna a nivel de Pueblo, hoy establecido en seis departamentos, y allí en 19 municipios del país, estas formas son: Tullpa, tradición oral, fiestas andinas, la Minga, casorios, velorios, alumbranzas, nacimientos entre otros espacios.

Estos momentos y dinámicas que se viven como Yanaconas han logrado mantenerse vigentes en muchos lugares de donde son oriundas la mayoría de los padres y madres mayores y mayoras que hoy tienen sus hijos e hijas en la ciudad de Popayán. 
Los círculos de la palabra en si se relacionan con estos temas de manera especial, están allí inmersos aportando desde el pensamiento propio mucho saber que estamos seguros contribuirá en el futuro de las próximas generaciones Yanakuna.

Las tulpas son espacios de vida entre los yanaconas y otros pueblos de América, es una de las formas del manejo del fuego para la preparación de sus alimentos y otras formas culturales. Alrededor de la tullpa se establecieron muchos fundamentos de vida familiar y comunitaria. Así mismo en este espacio los mayores y mayoras de manera constante al igual que los padres y madres en el hogar compartían sabiduría como palabras mayores, historias de vida, el idioma propio y otras anécdotas que con el paso del tiempo se convirtieron en leyes de la vida misma de los pueblos originarios.

La tullpa en este recorrer de la vida es una escuela, un lugar donde muchos ombligos de muchos wawas fueron enterrados. Se creía que enterrando los ombligos debajo de la tullpa los Yanakuna al crecer no se irían de la casa y menos de la comunidad. La tullpa además es un espacio de conocimiento, allí con el calor del fuego se arreglaba la matriz de las madres, también se sobaban lisiados, entre otras actividades de la vida Yanakuna. (Pensamiento Colectivo Yanakuna, 2015).

Los Yanaconas al igual que muchos de los pueblos originarios en el Abya Yala, han realizado sus conversatorios en forma circular, esto se desprende de fundamentos y principios adquiridos en los inicios del mundo Inca, puesto que el origen de este pueblo milenario hoy está muy claro que se originó en el Perú en lugar llamado YANA YAKU- Agua negra, desde allí se empieza una gran travesía de luchas historias de vida para trascender, se habla de que los yanaconas perdieron una guerra y que por ello pasaron a ser acompañantes de los Incas, el servicio consistía en realizar ciertas labores especiales teniendo en cuenta la capacidad y habilidad de los yanaconas. Muchos de ellos se los incluía en temas de guerra, otros en política, artesanos, sabios, consejeros, médicos o kuracas, otros en cambio no tenían habilidades y no realizan labor alguna para el Inca , así se explica en el reciente libro denominado "Hacia el Hatun Yana Marka-Pueblo Yanacona. (Pensamiento colectivo Yanacona 2014).

En este sentido la tradición oral es una forma de comunicar de generación en generación los logros, dificultades, sabidurías en todos los campos de la vida en una forma práctica que se basa en conocimientos experimentados en la práctica, así pues este mecanismo se mantiene vigente en los contextos de vida yanacona de muchos resguardos y cabildos aunque con un cierto grado de intención pero si con un mensaje cultural, muchos de estos ejercicios se viven en los campos de la medicina ancestral, pues los Yachak o sabios de la comunidad no escriben la forma de hacer un pegamento a la madre tierra, pues se viven en la práctica. (Pensamiento Colectivo Yanacona, 2014).

La tradición oral Yanakuna se establece en el compartir de la palabra alrededor de la tullpa u otros espacios de dialogo en donde se comparte comida propia, saberes 
propios. Es interesante tener en cuenta que en la vida Yanakuna la tradición oral se compartía en la familia, de abuelos a padres y madres y de padres y madres a sus hijos. Los métodos eran generalmente en las noches, puesto que durante el día se trabajaba, ya en la noche era el espacio en la cocina principalmente donde se daban los momentos para recordar y compartir la memoria ancestral. Muchos de las familias dentro de sus principios han mantenido aun proceso de compartir la palabra dando a conocer lo que sucede alrededor de la comunidad, el proceso político organizativo, la guardia, correctivos entre otros temas.

La tradición oral para el Yanakuna es además un espacio en donde se vivencian temáticas de antaño o de épocas anteriores en los campos de justicias propia, el manejo de la tierra, los usos y costumbres, cosmovisiónes, mingas, casería, , espantos, palabra mayor entre muchos otros aspectos, así mismo en la actualidad estos elementos se siguen revitalizando desde la tradición oral. Hoy los temas son distintos en campos de la gobernabilidad, la educación propia, la salud intercultural y así mismo muchos elementos que hacen parte del plan de vida. (Pensamiento colectivo Yanacona, 2015).

\section{Los Raymi (Fiestas Andinas)}

Los raymis son un espacio que se acoge a los indígenas Andinos en un proceso de celebración y agradecimiento a sus Dioses ancestrales, en este sentido desde el año 2002 en el territorio Yanakuna, ha generado la integración que se ha venido realizando con otros pueblos andinos en especial, los Kichwas de Ecuador y algunos encuentros con compañeros y compañeras caminantes Yanakuana y de otros pueblos hermanos del Ecuador, Perú, Bolivia y Argentina entre otros.

Aquí el cultivo y crianza ejerce una trascendencia importante, pues en los espacios que se tuvo, se realizó una siembra espiritual y hoy fruto de ese florecimiento esta e auge la celebración de estas fiestas andinas:

\section{Pawkar Raymi: (Fiesta del Despertar) Marzo 21}

Para los Yanakuna es una fiesta ancestral que se viene realizando desde el año 2002 en promedio. Se trata de establecer el pagamento a la madre tierra por la época del florecimiento, es un espacio que se prepara para las siembras, es época de iniciar los ciclos de siembra del maíz y otros productos que se establecen en las comunidades. Cultivos y semillas son preparados armónicamente y se ofrenda a la pacha mama o madre tierra pidiendo permiso para que el espíritu de las plantas y semillas fluya y traiga muy buenas cosechas. En este proceso tuvimos la oportunidad de llevar a cabo la celebración del Pawkar Raymi en un pequeño grupo de trabajo con mayores, niño y jóvenes a las afueras de la ciudad de Popayán el día 21 de marzo del presente año donde se compartieron importantes memorias y palabras de vida. 


\section{Inti Raymi: (Fiesta del sol-Año nuevo) Junio 21}

Es la fiesta del nacimiento del sol bebé, es un espacio que nos permite interactuar con un acontecimiento milenario en donde el sol nuevamente nace después de alumbrar un año completo, desde occidente se le denomina el solsticio de verano. En el inti Raymi hay unos preparativos especiales en donde se alistan las mejores semillas, las mejores ideas y proyectos, así mismos se hacen peticiones al sol bebe en temas de salud, sanaciones y todo proyecto de vida. Se ofrenda coca e inciensos llamándolo para que apoye y de la mejor luz y energía durante el año que se inicia cada 21 de junio.

\section{Killa Raymi: (Fiesta de la Luna) 21 de septiembre}

Es una fiesta a la mujer, es un espacio dentro del calendario andino Yanakuna en donde se da fuerza y revitalización a la mujer, al concepto de la complementariedad, es además un espacio para valorar, respetar y armonizar toda fuente de vida del género hembra. La fiesta de la luna como se le llamaría en Español permite establecer un equilibrio de energías, pues como el sol, que hace parte del genero macho ha dominado en su espacio de alumbramiento de luz y calor, necesita ser también dominado en otras palabras. Por ellos el Killa Raymi es la fiesta que armoniza y equilibra el sistema de vida en la pacha mama. Esta fiesta le permite a la mujer Yanakuna así mismo retomar energías y poner sus ideas para un año más de trabajo. En esta época se desarrollan algunas cosechas, puesto que luego de seis meses ya el maíz da sus mazorcas y es una época en donde hay maíz maduro con el que se prepara aku (maíz tostado y molido) muy útil para las faenas de trabajo.

\section{Kapak Inti Raymi: (Fiesta Mayor del Sol) Diciembre 21}

La fiesta del Kapak inti Raymi es la fiesta de celebración del máximo esplendor del sol, es la cúspide en donde el sol llega a alumbrar con mayor intensidad y por eso es una época para llenarse de la fuerza energética del inti tayta (padre sol). Los Yanakuna han venido desarrollando esta fiesta para compartir mucha palabra mayor con los caminantes y en si es una fecha en donde desde la madurez de nuestros mayores y mayoras se comparte la palabra sabia, la palabra mayor que se ha caminado con mucha experiencia durante los procesos del caminar Yanakuna. (Pensamiento Colectivo Yanacona, 2015).

\section{La minga Yanakuna}

La minga es un espacio de trabajo colectivo que se ha mantenido en el concepto de vida de los Yanaconas y así mismo es práctico aun en muchos espacios. La fortaleza de este uso y costumbre es la fuerza en la mano de obra. En este espacio es importante tener en cuenta que hay distintas dinámicas de una minga, hay mingas de trabajo de chakra (huerta), así mismo se ejercen mingas de pensamiento, de espiritualidad, de 
matrimonio, mingas de acompañamiento cuando un comunero parte al uku pacha (mundo interno-de abajo). En si son espacios que dinamizan los distintos círculos de la palabra en los Yanakuna del contexto de ciudad.

\section{Minga para celebrar un casorio o matrimonio}

Los casorios son una forma de los círculos de palabra, en ellos se vivencian en si muchos de los fundamentos de vida de los Yanaconas, llama la atención que aquí se comparten usos y costumbres, ritualidades, comidas propias. Muchos de los matrimonios anteriormente se expresa por Maximiliano Melenje Quinayás (abuelo del resguardo de Guachicono) que eran de mucho respeto, pues en la época de los años cincuenta por la colonización religiosa estos tenían una trascendencia de obligatoriedad y se hacían compromisos entre mayores, sin el consentimiento de los jóvenes. En si este proceso era toda una gran minga en donde todos los vecinos y amigos acudían a sembrar, arreglar la casa donde vivirían los nuevos casados.

Las mingas de matrimonio según el abuelo y mayor Maximiliano Melenje en su época de juventud de 1930 eran unos espacios donde concurría mucha gente Yanakuna, en su resguardo se vivenciaban estos espacios y eran los pocos espacios de distracción, pues el resto de los días era trabajo arduo. Estas actividades de integración detenían los procesos de siembra, cosecha $u$ otras actividades que se vivían en las frías tierras del resguardo ancestral indígena de Guachicono al sur del Cauca.

\section{Minga para acompañar la despedida de un Yanakuna que parte a otro espacio de vida}

Dentro del mundo Yanakuna catolizado antiguamente se realizaban procesos muy propios de la época en donde se creía que el espíritu iba al cielo, pero pese a toda esa dinámica colonizadora se incluían los ritos propios como ponerle comida o fiambre al difunto, así mismo su mejor herramienta, pues se creía que las personas llegarían a otro mundo a realizar unas labores idénticas a las realizadas en este espacio de vida convencional. Así que la minga de acompañamiento a este proceso de partida de los seres queridos ha contado con apoyo solidario de los yanaconas, se llevan alimentos y se comparte memoria, música de chirimía entre muchos otros procesos.

\section{Minga para acompañar y celebrar una alumbranza. (Se alumbran santos con velas)}

Las alumbranzas hacen parte de la tradición de los indígenas Yanaconas en el macizo Colombiano, pese a que este es un proceso que se implementó con la llegada de la religión Católica, cuenta con una gran connotación de tradiciones indígenas que se mantienen en los espacios que se comparten. De acuerdo a la consulta hecha los 
mayores expresan que anteriormente en las alumbranzas se compartía la unidad familiar, la religiosidad y así mismo juegos tradicionales, comida y bebidas propias.

Muchos de los procesos de enamoramiento se dieron en estos espacios, allí era el único lugar que los jóvenes podían al menos cruzar una que otra palabra, o cogerse las manos debajo de la ruana si había atracción. La rigurosidad de los padres y madres de la época de hace unos cincuenta años funcionó así.

Las alumbranzas se realizaban en espacios en donde se alumbraba a los santos patronos de las comunidades, unos como es el caso de San Sebastián realizaban estos ceremoniales al santo Patrono San Sebastián, como se le ha denominado por los creyentes o fieles.

En Guachicono un resguardo vecino las alumbranzas se realizaban en honor al niño de Chakilulun (huevos de pie) en Pancitará a la Virgen de las Mercedes y así sucesivamente el proceso variaba.

\section{Minga para acompañar y recibir un/una wawa (niño/a) recién nacido}

En la tradición Yanacona de los resguardos de Papallaqta y San Sebastián la voz mayor ha dejado la tradición que este proceso es de alta importancia. La llegada de un/una nuevo wawa a la comunidad representó todo una alegría, un espacio para cuidar a las madres y también un espacio en donde las parteras o sabedores en algunos casos los mismos padres.

Al nacer un wawa o niño(a) la placenta se preparaba con plantas y se daba un mensaje a ella para que guiara y construyera en el nuevo ser una persona comunitaria. Se enterraba luego a 20 metros de distancia de la casa en un espacio que diera con la el frente de la entrada principal de la casa o choza de paja. Allí se realizaba un hueco de 60 centímetros, seguidamente se echaba ceniza y rescoldo como le llaman los mayores a la brasa caliente.

Con otras plantas y aguardiente luego se echaba y salía un humo caliente, y justo allí se ponía la placenta con buen camino, con un mensaje para que sea llevado al corazón de la madre tierra. La dimensión era enterrarla a los treinta centímetros o sea a la mitad del orificio hecho para el ritual. Se dice que si no se hacía así podía suceder lo siguiente:

Enterrada muy abajo, los dientes salían muy pequeño en el wawa, muy encima enterrada la placenta, al wawa (bebé) le salían dientes muy grandes, por eso muchos ejemplos de malos procesos hechos son latentes en las comunidades. (Pensamiento colectivo obtenidos en círculos de palabra Yanakuna 2015). 
Todo los aspectos mencionados anteriormente hacen parte de la comunicación propia del pueblo Yanakuna en Colombia, el desarrollo de los distintos espacios de vida colectiva hacen que día tras día nos podamos encontrar en círculo de palabra y trascender hacia otros 500 años de lucha y resistencia hacia el retorno del tan aheleado buen vivir que buscamos en el caminar.

\section{Materiales y métodos}

\section{La Chakana, un camino metodológico para revitalizar el círculo de la palabra en la comunidad Yanakuna de Popayán}

Es una metodología ancestral en el pueblo Yanacona, este ejercicio trata de demostrar que desde la cosmovisión propia de este símbolo originario es interesante encontrar las respuestas a las hipótesis que se plantean en un determinado espacio o contexto de vida. Aquí existen cuatro caminos, desde ellos se establecen unos vínculos y entre ellos se enlazan para dar respuesta de forma armónica. Esta herramienta ancestral es de suma importancia dentro del proceso de la comunidad Yanakuna de Popayán, puesto que es volver a pensar como los mayores, es encontrarse con la esencia de la vida.

El concepto de la chakana viene de origen ancestrales milenarios, esta palabra se ha usado en nuestros territorios para llevar a los enfermos o muertos a un determinado espacio, el concepto tal vez hasta hace algunos años era desconocido, pues solo nos limitábamos a pronunciar su nombre y usar el instrumento cuando se requería.

En la actualidad dentro del proceso Yanacona que ha avanzado, gracias a caminantes que se han interesado en investigar y se llega a la conclusión que con la chakana se pueden abordar cuatro grandes caminos que son en si la esencia de la gente Yanakuna. Estos cuatro grandes caminos son: El territorio pedagógico, La Sabiduría ancestral, los Procesos Político Organizativos y la Producción de Conocimiento.

Estos caminos son la esencia para adelantar cualquier proceso de trabajo en el campo del conocimiento, y en si son elementos que todo Yanakuna desarrolla así desde su sentir, ver y actuar. Los procesos en si están en la mente, en el hablar, en el sentir y en el hacer de las personas que caminan como indígenas Yanakuna.

Estos cuatro grandes caminos dentro de esta investigación juegan un papel trascendental puesto que dan la guía para poder abordar distintas temático de la cultura y son de fácil entendimiento para revitalizar los procesos en los campos de la educación propia, los mismos ejercicios de la comunicación intercultural entre otros espacios. 


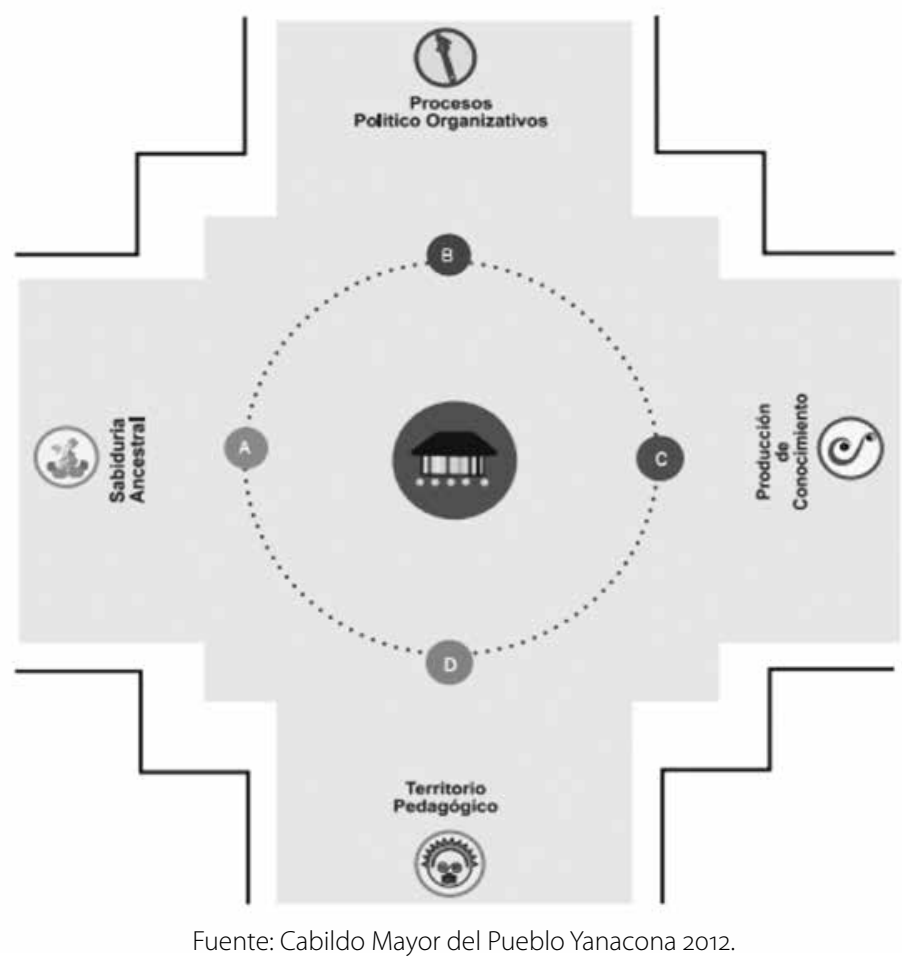

Frente a la chakana es posible desde el circulo de la palabra abordar cuatro círculos de palabra en donde en cada uno de los espacios se encontraran elementos muy significativos. Un breve ejemplo el circulo de la palabra con la Sabiduría ancestral en donde los mayores son los protagonistas nos posibilitaría conocer muchos procesos de realidades históricas, ciencia, tecnología, arte indígena entre muchos otros más conocimientos.

En el círculo de palabra en el camino de la Chakana denominado Procesos Político Organizativos es un espacio en donde con los guías Yanakuna, las autoridades, dinamizadores se puede conocer que se está pensando como pueblo, que avances se han tenido, como estamos pensando a otros 500 años entre muchos otros aspectos.

En el círculo de palabra en el camino de la Chakana denominado Producción de Conocimiento es una de las metas poder avanzar en la transformación de todo el saber indígena que se vive en la tullpa o circulo de pensamiento con la producción de materiales propios, entre ellos video documentales, programas radiales y escritos para compartir con la comunidad yanacona en la ciudad.

En el círculo de palabra en el camino de la Chakana denominado Territorio Pedagógico es una de las oportunidades más valiosas para desarrollar el saber en el contexto, allí es llegar a comprender que todo tiene energía, que nada es solo, que 
cada ser por pequeño que sea tiene unos lenguajes propios y unas formas y modos de comunicarse, es allí donde se puede aprender la verdadera esencia para un Yanakuna de contexto de ciudad.

\section{Resultados y discusión}

Este cultivo y crianza de sabidurías y conocimientos está proyectado a diez años y esta soñado para aportar en el proceso de la descolonización de nuestros compañeros y compañeras en el contexto de la ciudad de Popayán, así mismo es una herramienta que puede ser válida para que otras experiencias de vida Yanakuna en la ciudad puedan retomar algunos elementos y ponerlos a consideración para nutrir los ideales como pueblo.

Es también una de las proyecciones de esta propuesta como CCRISAC nutrir desde el punto de vista descolonizante otra forma de abordar la investigación, pues en la mayoría de los proyectos académicos a los que están acostumbrados nuestros egresados solo se limitan a dejar plasmado un documento que luego se archiva y no construye vida Yanakuna, esta propuesta por lo contrario es un mensaje de palabra mayor que motiva e incita a participar, a involucrarnos en la minga y hacer parte activa de la construcción de nuestra historia Yanakuna.

Considero que la ciudad es un espacio que aunque parece difícil cultivar y criar sabiduría es posible, son las voluntades y las didácticas propias las que pueden dar un toque mágico de cambio en nuestro proceso político organizativo y cultural.

Dentro de los acuerdos y compromisos, es dar a conocer este documento y hacerlo viable para que pueda ser leído y desglosado por los comuneros de la comunidad Yanakuna de la ciudad de Popayán. Es parte de los compromisos por contribuir al impacto de la educación en la comunidad, con idea y otras formas de construir conocimiento. El CCRISAC es un camino, una senda que se inicia en la vida de los Yanakuna. Personalmente, estoy totalmente seguro que esta propuesta de cultivo y crianza nos dará a futuro frutos maduros y positivos para trascender en el pasado y caminar hacia el futuro.

\section{Conclusiones}

La propuesta de Transformación que busca esta investigación desarrollada es aportar desde espacios descolonizantes desde el circulo de la palabra nuevas ideas y modos de compartir la palabra, hoy los pueblos indígenas y en especial nuestra comunidad Yanakuna tiene en su presente una nutrida serie de herramientas ancestrales que le pueden dar vida a los seis pilares del plan de vida, es así que desde el P.E.S.C.A.R, en donde los pilares Político, Educativo, Social, Cultural, Ambiental y de Relaciones 
Internas y Externas tendrá una oportunidad de nutrirse para trascender en otra era del conocimiento propio.

El saber ancestral de nuestro pueblo Yanakuna debe ser valorado y puesto en movimiento a travez de los círculos de la palabra. Las nuevas generaciones de nuestro pueblo tienen en si una responsabilidad cultural para que la siembra que han hecho sus los mayores en ellos, siga caminando. La palabra mayor no es nuestra , es ancestral, viene desde la transmisión espiritual, hoy los caminantes son los transmisores de ella y en esencia el circulo de la palabra es el espacio que permite que ella renazca, tenga vida, camine, sea sembrada y luego germine en otros espacios de vida.

\section{Lista de referencias}

Achury, F. (2015). Círculo de la Palabra, Tradición Ancestral. Recuperado en: http://www.chamanismoparatodos.com/2015/o6/o2/circulo-de-la-palabra-tradicion-ancestral/

Agencia de Noticias, UNC. (12 Abril del 2016). "Círculos de la palabra”, un juego de relatos indígenas. Universidad Nacional de Colombia, (490). Recuperado en: http:// agenciadenoticias.unal.edu.co/detalle/article/circulos-de-la-palabra-un-juegode-relatos-indigenas.html

CRIC (2011). Sistema Educativo Indígena Propio SEIP - CRIC -PEBI Primer documento de Trabajo.

(2012). Legislación Indígena. Consejo Regional Indígena del Cauca.

(2015a). Cartilla Familia, Territorio y Comunidad. Consejo Regional Indígena del Cauca.

(2015b). Investigación y Pensamiento Indígena. Consejo Regional Indígena del Cauca.

Estudios de Cultura e Historia Andina: Hacia el Hatun Yana Marka - Historia del Macizo Colombiano. Primera Edición 2014.

Oralidad en Latinoameríca (21 Diciembre, 2010). Memoria Oral: la importancia de la palabra. Recuperado en: https://oralidadlatinoamericana.wordpress. com/2010/12/21/memoria-oral-la-importancia-de-la-palabra/ 2010. 